\title{
FGFR1 is a Potential Therapeutic Target in Neuroblastoma
}

\section{Flora Cimmino}

University of Naples Federico II, CEINGE Biotecnologie Avanzate

Annalaura Montella

Università degli Studi di Napoli Federico II

Matilde Tirelli

Università Degli Studi di Milano

Marianna Avitabile

University of Naples Federico II, CEINGE Biotecnologie Avanzate

Vito Alessandro Lasorsa

University of Naples Federico II, CEINGE Biotecnologie Avanzate

\section{Feliciano Visconte}

University of Naples Federico II, CEINGE Biotecnologie Avanzate

\section{Sueva Cantalupo}

Università degli Studi di Napoli Federico II

Teresa Maiorino

Università degli Studi di Napoli Federico II

Biagio Angelis

Bambino Gesù Children's Hospital IRCCS

Martina Morini

IRCCS Istituto Giannina Gaslini

\section{Aurora Castellano}

Bambino Gesù Children's Hospital IRCCS

Franco Locatelli

Bambino Gesù Children's Hospital IRCCS

Mario Capasso ( $\sim$ mario.capasso@unina.it)

Università degli Studi di Napoli Federico II

Achille lolascon

Università degli Studi di Napoli Federico II

\section{Research Article}

Keywords: FGFR1, metastasis, mutation, neuroblastoma, targeted therapy

Posted Date: February 16th, 2022

DOI: https://doi.org/10.21203/rs.3.rs-1353035/v1

License: (9) (1) This work is licensed under a Creative Commons Attribution 4.0 International License. Read Full License 


\section{Abstract}

Background: FGFR1 regulates cell-cell adhesion and extracellular matrix architecture and acts as oncogene in several cancers. Potential cancer driver mutations of FGFR1 occur in neuroblastoma NB, a neural crest-derived pediatric tumor arising in sympathetic nervous system but so far, they have not been studied experimentally. We investigated the driver-oncogene role of FGFR1 and the implication of N546K mutation in therapy-resistance in NB cells.

Methods: Public datasets were used to predict the correlation of FGFR1 expression with NB clinical outcomes. Whole genome sequencing data of 19 paired diagnostic and relapse NB samples were used to find somatic mutations. In NB cell lines, silencing and transient overexpression of $F G F R 1$ by short hairpin RNA were performed to evaluate the effect of the found mutation by cell growth, invasion and cologenicity assays. HEK293, SHSY5Y and SKNBE2 were selected to investigate subcellular wildtype and mutated protein localization. FGFR1 inhibitor (AZD4547), alone or in combination with PI3K inhibitor (GDC0941), was used to rescue malignant phenotypes induced by overexpression of FGFR1 wildtype and mutated protein.

Results: High FGFR1 expression correlated with low relapse-free survival in two independent NB gene expression datasets. In addition, we found the somatic mutation N546K, the most recurrent point mutation of FGFR1 in all cancers and already reported in NB, in one out of 19 matched primary and recurrent tumors. Loss of FGFR1 function attenuated invasion and cologenicity in NB cells, whereas FGFR1 overexpression enhanced oncogenicity. The overexpression of FGFR1 ${ }^{\text {N546K }}$ protein showed a higher nuclear localization compared to wildtype protein and increased cellular invasion and cologenicity. Moreover, N546K mutation caused the failure in response to treatment with FGFR1 inhibitor by activation of ERK, STAT3 and AKT pathways. The combination of FGFR1 and PI3K pathway inhibitors was effective in reducing the invasive and colonigenic ability of cells overexpressing FGFR1 mutated protein.

Conclusion: FGFR1 is an actionable driver oncogene in NB and a promising therapy may consist in targeting FGFR1 mutations in patients with therapyresistant NB.

\section{Background}

Neuroblastoma (NB) arises from malignant transformation of neural crest-derived precursors of the peripheral sympathetic nervous system and occurs in $5 \%$ of pediatric cancers in patients younger than 19 years ${ }^{1}$. The discovery of genomic markers such as MYCNamplification, $17 \mathrm{q}$ gain, $11 \mathrm{q}$ and $1 \mathrm{p} 36$ deletions has greatly improved risk stratification and prognosis of younger affected patients ${ }^{2}$. Instead, different genomic aberrations characterize NB in late childhood and adolescence, often showing $19 \mathrm{p}$ loss and 1q gain ${ }^{3}$. Additionally, genome-wide association studies ${ }^{4 ; 5}$ and candidate gene approaches ${ }^{6-8}$ have identified multiple DNA polymorphisms influencing NB susceptibility and clinical phenotype that may represent novel potential outcome predictors $9 ; 10$.

High-risk NBs comprise nearly half of all NBs and have a long-term survival of $<50 \%$, with almost $60 \%$ of affected children being non-responsive to advanced treatments and dying due to relapse ${ }^{11}$. More recently, it has been shown that, among high-risk, gene expression-based signatures can identify children with higher risk disease who would benefit from new and more aggressive therapeutic approaches ${ }^{12-14}$. Next generation sequencing studies have documented a paucity of mutations in recurrently affected genes in primary NB and an increase of "potentially actionable" mutations in relapse ${ }^{15-17}$. In primary tumors mutations in ALK, ATRX and TERT have been identified as the most frequent genetic abnormalities ${ }^{18-21}$, whereas in relapse an increased number of damaging or deleterious mutations in cell motility and cell survival pathways (e.g. PI3K/AKT/mTOR, MAPK or noncanonical Wnt pathways) has been reported 22 . Moreover, the selection of subclones with driver mutations in the RAS-MAPK pathway between the primary and the relapse tumors may occur as resistance mechanisms ${ }^{16}$, but more research is needed to unravel the underlying causes. These data suggest that NB undergoes substantial mutational evolution during therapy and that relapsed disease is more likely to be driven by a targetable oncogenic pathway. Recently, we reported that somatic noncoding variants located in regulatory DNA elements specifically activated in NB tumors can contribute to tumorigenesis ${ }^{23}$

Fibroblast growth factor (FGF) signaling cascades throught FGF receptor 1 (FGFR1) leads to the activation of MAP kinases. Alterations in FGFR1 have been reported in $3.63 \%$ of all cancers, with breast carcinoma, non-small cell lung carcinoma, colorectal adenocarcinoma, malignant glioma and ovarian neoplasms showing the greatest prevalence of abnormalities ${ }^{24}$. The most common alterations in FGFR1 are amplifications (2.34\%), point mutations (1.20\%) and gene loss $(0.33 \%)^{24}$. Among the point mutations, the most recurrent one is N546K $(0.14 \%)^{24}$, that has been found in NB primary ${ }^{15 ; 18}$ and in the paired relapsed tumors ${ }^{15 ; 16}$. Moreover, in addition to the already reported single relapsed NB case ${ }^{15}$, N546K mutation has also been recently reported in 6 patients ${ }^{25}$. Specifically, N546K represents an activating mutation that alters FGFR1 auto-phosphorylation ${ }^{26}$, resulting in an increase of kinase activity and malignant transformation in Ewing sarcoma and brain tumors ${ }^{27-31}$.

FGFR contitutes a promising druggable target in cancer and different approaches for inhibiting FGFR, including selective and nonselective FGFR smallmolecule tyrosine kinase inhibitors, monoclonal antibodies against FGFRs and FGF ligand traps are under investigation in several phase I/II clinical trials ${ }^{32}$

The aim of this study was to characterize FGFR1 as NB cancer-driver gene and to evaluate its role as theraupetic target with in vitro studies.

\section{Methods}

\section{Microarray-KAPLAN SCAN}

R2 web tool (http://r2.amc.nl) was used to predict the association of FGFR1 expression with survival of patients with NB. In brief, for each gene, R2 calculates the optimal cut-off in the expression level to divide the patients into 'good' and 'bad' prognosis cohorts. Samples within a dataset are sorted according to the expression of the investigated gene and are divided into two groups. All the cut-off expression levels and their resulting groups are analyzed according to patient survival. For each cut-off level and grouping, the log-rank significance of the projected survival is calculated. The best $P$ value and the corresponding 
cut-off are selected. The cut-off level is reported and was used to generate the Kaplan-Meier curves. These depict the log-rank significance (raw $P$ ) as well as the $P$ value corrected for multiple testing (Bonferroni correction) of the cut-off levels for each gene. Kaplan scan analysis was performed to estimate the overall survival and relapse-free survival according to FGFR1 in the three microarray datasets: the Seeger dataset that included 102 International NB Staging System stage 4 patients without MYCN amplification; the Versteeg dataset that included 88 patients with different clinical characteristics and the Asgharzadeh TARGET dataset that includes 247 patients.

\section{Whole genome sequencing}

In-house WGS data. WGS of 10 normal-primary-relapse NB sample triplets was performed on an Illumina HiSeq1500 platform. The paired-end sequencing produced $150 \mathrm{bp}$ long reads. Alignment files were obtained by mapping reads versus GRCh37/hg19 reference genome assembly. Somatic SNVs and INDELs were detected with MuTect ${ }^{33}$ and Strelka ${ }^{34}$, respectively.

Publicly available WGS data (Target). We obtained access to WGS of NB from the TARGET project ${ }^{35}$ (Accession: phs000218.v21.p7; Project ID: \#14831) and included, in our analysis, 9 normal-primary-relapse NBs for which somatic variants were available. The functional annotation of somatic variant calls was performed with ANNOVAR ${ }^{36}$ and FunSeq2 ${ }^{37}$.

\section{Copy number variation analysis}

We evaluated the copy number (CN) status of FGFR1 in NB patients of the TARGET-NB project. Open access level 3 (L3) copy number segmentation data of 381 NB samples ${ }^{38}$ were downloaded from NIH Office of Cancer Genomics website (https://ocg.cancer.gov/). The R - Bioconductor "copynumber" package ${ }^{39}$ was implemented to estimate CN status starting from Log R Ratio (LRR) and B Allele Frequency (BAF) information. For both data sets we set stringent cutoffs to call CN changes: CN losses were called for LRR below -0.42 ( $C N<1.5)$; normal LRR values were between -0.42 and 0.58 ( $C N$ ranging from 1.5 to 3 ); a $C N$ gain was called if LRR was between 0.58 and 1.3 (CN ranging from 3 to 4.9 ); we called amplification for LRR greater than or equal to 1.3 (CN $\geq 4.9$ ). RefSeq FGFR1 transcript variant 1 (NM_023110) genomic coordinates were taken from UCSC genome browser (http://genome-euro.ucsc.edu/) and used to search for the presence of CNVs in samples of the abovementioned data sets.

\section{Cell culture}

The human SHSY5Y and HEK293 cells were grown in Dulbecco's modified Eagle's medium; SKNBE2 cells were grown in Dulbecco's modified Eagle's medium F12. Both cell lines were supplemented with $10 \%$ heat-inactivated fetal bovine serum (Sigma), $1 \mathrm{mM} \mathrm{L-glutamine,} \mathrm{penicillin} \mathrm{(100} \mathrm{U/ml)} \mathrm{and} \mathrm{streptomycin} \mathrm{(} 100$ $\mu \mathrm{g} / \mathrm{ml}$ ) (Invitrogen), at $37^{\circ} \mathrm{C}$, under $5 \% \mathrm{CO} 2$ in a humidified atmosphere. AZD4547 and GDC0941 were diluted in DMSO at $10 \mathrm{mM} / \mathrm{ml}$ and stored at $-20^{\circ} \mathrm{C}$ until use. The inhibitors were diluted at $0.1 \mu \mathrm{M}$ and $1 \mu \mathrm{M}$ in the culture medium without serum.

\section{Production of lentiviral particles and infection of cell lines}

To knock-down FGFR1 expression, the pGIPZ lentiviral shRNAmir that targets human FGFR1 were purchased from Open Biosystems (Thermo Fisher Scientific, Inc.). We used two different shRNAs for FGFR1 gene. The shRNAs against FGFR1 were: shFGFR1\#A (V3LHS_644622) and shFGFR1\#B (V3LHS_634642). A non-silencing pGIPZ lentiviral shRNAmir was used as the control (RHS4346). HEK293T were transfected using $10 \mu \mathrm{g}$ shRNA plasmid DNA, $30 \mu \mathrm{l}$ TransLentiviral Packaging Mix (OpenBiosystem), and $25 \mu \mathrm{l}$ TrasFectin (BioRad), in 10-mm plates. The supernatants (10 ml per condition) were harvested after $24 \mathrm{~h}$, centrifuged at a low speed to remove cell debris, and filtered through $0.45-\mu \mathrm{m}$ filters. Cells transduction was performed as previously described ${ }^{40}$.

\section{Western blotting}

Cell pellets were resuspended and lysed in RIPA buffer (50 mM Tris- $\mathrm{HCl}, \mathrm{pH} 7.5,150 \mathrm{mM} \mathrm{NaCl}, 1 \%$ Triton X-100, 10\% glycerol, and completed with protease and phosphatase inhibitors cocktail (ThermoScientific). Total proteins extracts concentrations are determinated by the Bradford assay (Bio-Rad). Cytosol and nucleus protein fractions were obtained as previously described ${ }^{41}$.

After 1 hour blocking with $5 \%$ non-fat dried milk (EuroClone) or bovine serum albumin (SERVA) in TBS with $0.1 \%$ Tween (TBS-T), the membranes were incubated with primary antibodies at $4^{\circ} \mathrm{C}$ overnight. Primary antibodies used: anti-pFGFR1 (06-1433, Millipore), anti-FGFR1 (Abcam ab137765), anti-pSTAT3 (D3A7, Cell Signaling), anti-STAT3 (06596, Millipore), anti-pAKT1 (ab81283; Abcam), anti-AKT1 (ab32505; Abcam), anti-pERK1/2 (ab32538; Abcam), antiERK1/2 (ab17942; Abcam), anti t-GFP (TA150041, Origene) and $\beta$-actin (Sigma, A5441). After membrane incubation with horseradish-peroxidase-conjugated anti-rabbit secondary antibody (Immuno Reagents), the positive bands were visualized using the ECL kit SuperSignalTM West Pico PLUS Chemiluminescent Substrate (Thermo Scientific) as previously shown ${ }^{42}$.

\section{Real-time RT-PCR}

Total RNA extraction using TRIzol LS Reagent (Invitrogen) and cDNA retrotranscription using the High-Capacity cDNA Reverse Transcription Script (Applied Biosistem) was performed according to the manufacturer protocol. Specific primers for FGFR1 (Forward: 5'-GCTAAAGCACATCGAGGTGAATG-3'; Reverse: 5'TCTCTTTGTCGGTATTAACTCC-3') and $\beta$-Actin (Forward: 5'- CGTGCTGCTGACCGAGG-3'; Reverse: 5'- GAAGGTCTCAAACATGATCTGGGT- 3') were designed by PRIMEREXPRESS software (Applied Biosystems). Real-time PCR was performed using SYBR Green PCR Master Mix (AppliedBiosystems) in the7900HT Fast Real-Time PCR System (Applied Biosystems). The experiments were carried out in triplicate for each data point. Relative gene expression was calculated using the 2- $\Delta \mathrm{CT}$ method, where the $\Delta \mathrm{CT}$ was calculated using the differences in the mean CT between the selected genes and the internal control ( $\beta$-actin).

\section{Cell viability assay}


Cells were seeded as six replicates into $96-$ well plates at a density of $10 \times 10^{3}$ cells per well. Cell viability is measured by measuring metabolic conversion (by viable cells) of the dye MTT [3-(4,5-dimethylthiazol-2I)-2, 5-diphenyltetrazolium bromide], according to the manufacture protocol (Promega) as previously described ${ }^{40}$ - For treatment with drugs, after cells attached to well, culture medium containing different drugs concentrations was added to cells. Cell viability was measured at $24 \mathrm{~h}, 48 \mathrm{~h}$ and $72 \mathrm{~h}$. Inhibitory concentration $\left(\mathrm{IC}_{50}\right)$ values were calculated uding nonlinear best fit regression analysis by Excel.

\section{Invasion assay}

Transwell chambers (Corning) were pre-coated with matrigel (BD Biosciences) at $37^{\circ} \mathrm{C}$ for 30 minutes. 80.000 cells resuspended in $350 \mu$ l serum-free medium were added to the upper compartment, and to the lower chamber was added $750 \mu$ LMEM containing $10 \%$ FBS, heat inactivated fetal bovine serum (Sigma), 1 $\mathrm{mM} \mathrm{L-glutamine,} \mathrm{penicillin}(100 \mathrm{U} / \mathrm{ml})$ and streptomycin $\left(100 \mathrm{\mu g} / \mathrm{ml}\right.$ ) (Invitrogen). Then the cells were incubated for $24 \mathrm{~h}$ at $37^{\circ} \mathrm{C}$ with $5 \%$ CO2. Transwell chambers were removed from the 24 plate and cells that have migrated were stained as previously described ${ }^{43}$. The invading cells were counted using the LeicaApplicationSuite/AF software and a DMI4000B microscope (Leica Mycrosystem). Chamber photos were acquired with 10x objective.

\section{Colony formation assay in soft agar}

The colony formation assay was performed to analyze anchorage-independent cell growth. Two hundred thousand cells were plated in $0.35 \%$ agar on a bottom layer of $1 \%$ agar in the 35 -mm dishes of 6 -well plates (Corning). The plates were incubated at $37^{\circ} \mathrm{C}$ for 4 weeks, and then stained with $0.01 \%$ crystal violet. Colonies with 20 cells or more were counted using the LeicaApplicationSuite/AF software and a DMI4000B microscope (Leica Mycrosystem) with 10x objective. The means and standard deviations were calculated from three independent experiments.

\section{Site-directed mutagenesis.}

Site-directed mutagenesis was performed on plasmid containing the coding sequence of human FGFR1 (RG202080, Origene) using a PCR-based strategy through KAPA HiFi Hot Start DNA polymerase (KAPA BIOSYSTEMS, London, United Kingdom). To introduce FGFR1 missense mutation N546K, primers were designed by the tool Primer-BLAST in web. Reaction mixture contained 0.5 KAPA HiFi HotStart DNA Polymerase, $300 \mu \mathrm{M}$ M KAPA dNTP Mix, $0.3 \mu \mathrm{M}$ forward and reverse primers, $1 \times$ expand 5X KAPA HiFi Fidelity Buffer and 50 ng plasmid DNA as template. Ultimately, PCR reaction was performed in the following conditions: $95 \circ \mathrm{C}$ for $5 \mathrm{~min} ; 35$ cycles of: $98 \circ \mathrm{C}$ for $20 \mathrm{~s}, 66 \circ \mathrm{C}$ for $15 \mathrm{~s}$ and $72 \circ \mathrm{C}$ for $90 \mathrm{~s}$; and finally, $72 \circ \mathrm{C}$ for 5 min. Product was treated with $1 \mathrm{U}$ Dpnl (NEB, USA) for 1 hour at $37^{\circ} \mathrm{C}$, and heat - inactivated at $80^{\circ} \mathrm{C}$ for 20 minutes. The new vector was analyzed by electrophoresis on $0,8 \%$ agarose gels and sequenced for verification.

\section{Cells transfections}

HEK293, SHSY5Y and SKNBE2 cells were seeded at a density of 250.000 cells/well in a 6-well plate and transfected with $2.5 \mu \mathrm{g}$ of pCMV6 empty vector or pCMV6 expressing FGFR $1^{\text {wt }}$ protein or pCMV6 expressing FGFR1 ${ }^{\text {N546K }}$ protein and $3 \mu$ l of TransFectinTM Lipid Reagent (Bio-Rad). Transiently transfected cells were subsequently starved in serum-free medium for $4 \mathrm{~h}$ and were harvested after 48 hours.

\section{ImageStream ${ }^{\mathrm{X}}$ Mark II Flow Cytometer acquisition and data analysis}

Cells were fixed in 4\% paraformaldehyde (10 min), permeated with $0.2 \%$ Triton X-100 (15 min), and blocked with $1 \%$ bovine serum albumin ( 30 min). The antiFGFR1 primary antibody (ab824, Abcam) was incubated for 90 min, and the AlexaFluor 647 goat anti-rabbit (A27040, Invitrogen) secondary antibody for 45 min. Then, cells were incubated with DAPI (Sigma) for 10 min to stain nuclei. A filter of $30 \mu \mathrm{m}$ was used to remove cells aggregates. ImageStream ${ }^{X}$ Mark II Flow Cytometer (EMD Millipore) was used to acquire single cells images at $60 x$ magnification. The acquired raw image file (.rif) contained among 500 and 2000 events (10-30 events per second). The analysis of single cells fluorescence intensity and nucleus diameter was performed by using IDEAS software (version 6.2.64.0). To consider only single cells a dot plot showing area versus aspect ratio (AR) was created. To estimate FGFR1 intensity in nuclear region we generated a morphology mask that defined nucleus stained by DAPI and to measure the fluorescent signal in nuclear area.

\section{Immunofluorescence confocal microscopy}

At 48h post transfection, HEK293, SHSY5Y and SKNBE2 cells were seeded on polilysine coated glass coverslips (Microtech S.R.L) overnight. Coverslips were fixed in $4 \%$ paraformaldehyde (10 min), permeated with $0.2 \%$ Triton-100 (15 min) and then blocked in 1\% bovine serum albumin (30 min). The anti-FGFR1 primary antibody (ab824) was incubation for $90 \mathrm{~min}$. Coverslips were then incubated in goat anti-Mouse IgG (H+L) secondary antibody Alexa Fluor 546 (Invitrogen A-11030) for 45 min. To stain coverslips were incubated with DAPI (Sigma) for 10 min. Slides were mounted with Mowiol ${ }^{\circledR} 4-88$ (Sigma-Aldrich, 81381) and visualized using a Leica TCS SP8 STED 3X confocal microscope (Leica Microsystems CMS GmbH).

\section{Neuropheres assay}

Neurospheres formation assay was performed in serum free medium containing half mixture of F12 and DMEM Low Glucose, supplemented with EGF 20 $\mathrm{ng} / \mathrm{mL}$, bFGF $40 \mathrm{ng} / \mathrm{mL}$, 2\% B27 (Gibco, ThermoFisher Scientific) and 1\% L-glutamine/penicillin-streptomycin. Cells were plated in the 35-mm dishes of 6-well plates (Corning). The plates were incubated at $37^{\circ} \mathrm{C}$ for three days following the cells seeding in serum-free medium. Spheres were observed and photographed under LeicaApplicationSuite/AF software and a DMI4000B microscope (Leica Mycrosystem). Chamber photos were acquired with 10x objective.

\section{Statistical analysis}


The differences between the groups were analyzed using unpaired student's $t$-test. Probability values $<0.05$ were statistically significant. * $p$-value $\leq 0.05, * \star p$ value $\leq 0.01, * \star *$ value $\leq 0.001$.

\section{Results}

\section{FGFR1 expression is associated with bad clinical outcomes in NB patients}

The association of FGFR1 expression with clinical outcomes was evaluated in three datasets that are deposited in the R2 microarray web tool (http://hgserver1.amc.nl/cgi-bin/r2/main.cgi): the Seeger dataset (102 patients with high-risk NB); the Versteeg dataset (88 patients) and the Asgharzadeh TARGET dataset (247 patients). Kaplan-Maier analysis showed that higher FGFR1 expression was significantly associated with inferior relapse-free survival in Seeger dataset $\left(p\right.$-value $\left.=3.1 \times 10^{-5}\right)$ and in Versteeg dataset $(p$-value $=0.057)$. In contrast, correlation of $F G F R 1$ to overall survival was not significant in Asgharzadeh TARGET dataset $(p$-value $=0.061)$ and in Versteeg dataset $(p$-value=0.118) $($ Fig. $1 \mathrm{~A})$.

FGFR1 expression analysis in a dataset of 11 primary and 7 relapsed tumors showed a higher FGFR1 expression in relapsed NB samples without reaching the significance level ( $p$-value=0.28), probably due to the limited number of samples (Fig. 1B).

Finally, we observed that FGFR1 mRNA levels in metastatic xenograft tumors were higher than those of NB primary tumors $(p$-value $<0.001)$ but were similar to those of embryonic cells and neuronal crest cells (Fig. 1C).

\section{FGFR1 somatic mutations and copy number variations in NB patients}

We analyzed whole genome sequencing (WGS) data at FGFR1 locus (including 50 kb surrounding regions) from 19 paired diagnostic and relapse NBs. WGS data from 10 samples were obtained in our laboratory whereas 9 were downloaded from TARGET project repository.

We found the hotspot mutation FGFR $1^{N 546 K}$ in one tumor at diagnosis and relapse (Table 1). No other putative coding pathogenic mutations were found. We also wanted to investigate potential pathogenic function of noncoding point mutations. To this purpose, we annotated each mutation with DNase I hypersensitive sites, known to define active regulatory DNA elements, in SKNSH NB cells (ENCODE data). No potential pathogenic mutations located in DNA regulatory sites were found (Table 1).

Since FGFR1 amplifications have been associated with other cancers, we analyzed copy number variations in a public dataset of 381 NBs. No significant amplification of FGFR1 was found (Fig. S1).

Table 1. Coding and noncoding somatic mutations found at FGFR1 locus in 21 primary-relapse pairs NB tumors analyzed by whole genome sequencing. 


\begin{tabular}{|c|c|c|c|c|c|c|c|c|c|c|}
\hline $\begin{array}{l}\text { Sample } \\
\text { ID }\end{array}$ & Type & Position/Change & Location & Gene & $\begin{array}{l}\text { Amino } \\
\text { acid } \\
\text { change }\end{array}$ & Band & $\begin{array}{l}\text { CADD } \\
\text { score }\end{array}$ & $\begin{array}{l}\text { COSMIC } \\
\text { ID }\end{array}$ & SNP ID & $\begin{array}{l}\text { ENCODE } \\
\text { annotation }\end{array}$ \\
\hline TR008 & $\mathrm{R}$ & chr8:38227325:G>T & intronic & WHSC1L1 & . & $8 p 11.23$ & 2,48 & . & . & Enhancer ${ }^{b}$ \\
\hline TR008 & $\mathrm{R}$ & chr8:38236248:C>G & intronic & WHSC1L1 & . & $8 p 11.23$ & 1,78 & . & . & . \\
\hline TR008 & $\mathrm{P}$ & chr8:38246999:C>G & intronic & LETM2 & . & $8 p 11.23$ & 1,77 & . & . & . \\
\hline TR008 & $\mathrm{R}$ & chr8:38246999:C>G & intronic & LETM2 & . & $8 p 11.23$ & 1,77 & & . & . \\
\hline TR001 & $\mathrm{P}$ & chr8:38267939:A>C & downstream & FGFR1,LETM2 & . & $8 p 11.23$ & 1,03 & . & . & TFP(CTCF) \\
\hline SP_2_T & $\mathrm{P}$ & chr8:38268616:C>A & downstream & FGFR1 & . & $8 p 11.23$ & 3,58 & & & TFP(CTCF) \\
\hline TR003 & $\mathrm{R}$ & chr8:38273003:A>T & intronic & FGFR1 & . & 8p11.23 & 2,25 & . & . & . \\
\hline PATNKP & $\mathrm{P}$ & chr8:38274849:G>T & exonic & FGFR1 & N457K & $8 p 11.23$ & 29,70 & YES ${ }^{a}$ & rs779707422 & . \\
\hline PATNKP & $\mathrm{R}$ & chr8:38274849:G>T & exonic & FGFR1 & N457K & 8p11.23 & 29,70 & YES $^{a}$ & rs779707422 & . \\
\hline TR007 & $\mathrm{P}$ & chr8:38282676:A>T & intronic & FGFR1 & . & $8 p 11.23$ & 2,96 & . & . & DHS(MCV-1) \\
\hline TR001 & $P$ & chr8:38288403:G>C & intronic & FGFR1 & . & 8p11.23 & 0,22 & . & . & $\begin{array}{l}\text { DHS(MCV-2); } \\
\text { TFP(SMARCC }\end{array}$ \\
\hline TR008 & $P$ & chr8:38295809:T>C & intronic & FGFR1 & . & 8p11.23 & 0,23 & . & rs975858205 & . \\
\hline PAUDDK & $\mathrm{P}$ & chr8:38296890:T>A & intronic & FGFR1 & . & $8 p 11.23$ & 17,63 & . & . & . \\
\hline TR003 & $\mathrm{P}$ & chr8:38301604:T>G & intronic & FGFR1 & . & 8p11.22 & 2,88 & . & rs947373873 & . \\
\hline PATNKP & $\mathrm{P}$ & chr8:38311785:C>G & intronic & FGFR1 & . & $8 p 11.22$ & 2,90 & . & . & \\
\hline PATNKP & $\mathrm{R}$ & chr8:38311785:C>G & intronic & FGFR1 & . & $8 p 11.22$ & 2,90 & . & . & . \\
\hline TR008 & $\mathrm{R}$ & chr8:38319864:G>A & intronic & FGFR1 & . & $8 p 11.22$ & 0,75 & . & . & . \\
\hline TR007 & $\mathrm{R}$ & chr8:38324367:G>A & intronic & FGFR1 & ${ }^{\circ}$ & 8p11.22 & 9,06 & . & . & $\begin{array}{l}\text { Enhancerb; } \\
\text { TFP(SIN3Á, } \\
\text { TAF7, TCF12, } \\
\text { YY1) }\end{array}$ \\
\hline TR008 & $\mathrm{P}$ & chr8:38337889:A>G & intergenic & FGFR1(dist=11537) & . & 8p11.22 & 3,92 & & & . \\
\hline TR008 & $\mathrm{R}$ & chr8:38338780:A>G & intergenic & FGFR1(dist=12428) & . & 8p11.22 & 18,68 & . & . & DHS(MCV-2) \\
\hline TR008 & $\mathrm{R}$ & chr8:38338784:C>G & intergenic & FGFR1(dist=12432) & . & 8p11.22 & 17,75 & & rs201380585 & DHS(MCV-2) \\
\hline TR006 & $\mathrm{R}$ & chr8:38349608:A>G & intergenic & FGFR1(dist=23256) & . & 8p11.22 & 0,61 & . & . & . \\
\hline TR006 & $\mathrm{R}$ & chr8:38350422:C>G & intergenic & FGFR1 (dist=24070) & . & 8p11.22 & 10,82 & . & . & . \\
\hline TR008 & $\mathrm{P}$ & chr8:38350507:A>C & intergenic & FGFR1(dist=24155) & . & 8p11.22 & 0,03 & . & . & . \\
\hline TR006 & $\mathrm{P}$ & chr8:38350642:T>C & intergenic & FGFR1(dist=24290) & . & $8 p 11.22$ & 0,04 & . & . & . \\
\hline PATNKP & $\mathrm{R}$ & chr8:38357592:C>A & intergenic & FGFR1(dist=31240) & . & $8 p 11.22$ & 4,03 & . & . & . \\
\hline TR006 & $\mathrm{R}$ & chr8:38369276:A>C & UTR3 & C8orf86 & . & $8 p 11.22$ & 0,45 & . & rs565928745 & . \\
\hline
\end{tabular}

aID=COSM3670398,COSM1284966,COSM1284968,COSM1284967,COSM19176;OCCURENCE=5(central_nervous_system),1(autonomic_ganglia)

P: Primary; R: Relapse.

${ }^{\mathrm{b}}$ Segway/ChromHMM-predicted enhancer.

TFP: transcription factor binding peak.

DHS: DNase I hypersensitive sites.

MCV-1, MCV-2: Cell lines of the ENCODE catalog.

\section{FGFR1 silencing impairs cells growth, invasion and colonigenicity in NB cells}

We investigated the role of FGFR1 in two NB cell lines: SHSY5Y MYCN non-amplified and SKNBE2 MYCN-amplified cells. 
We transduced SHSY5Y and SKNBE2 cells by lentiviral vectors encoding two independent short hairpin (sh)RNAs targeting FGFR1 (shFGFR1\#A and shFGFR1\#B) and a control shRNA (shCTR). Silencing efficiency was determined by western blotting and real time PCR (RT-PCR) (Fig. 2A).

Cell viability of both SHSY5Y and SKNBE2 shFGFR1 (shFGFR1\#A and shFGFR1\#B) significantly decreased compared to cell viability of shCTR at 48 and 72 hours ( $p$-value $\leq 0.05$ ) (Fig. 2B), suggesting that FGFR1 silencing impaired NB cell proliferation and cell growth.

Similarly, FGFR1 silencing affected NB cell ability to migrate through a matrigel-coated membrane (Fig. 2C and S2A) and the anchorage-independent growth, as shown by soft agar assay (Fig. 2D and S2B). Hence, colony numbers and invading cell numbers in shFGFR1 cells significantly decreased compared to shCTR cells in both SHSY5Y and SKNBE2 cell lines.

\section{FGFR1 ${ }^{N 546 K}$ exhibits a nuclear localization}

FGFR1 is constitutively found in cell membrane, cytoplasm and nucleus ${ }^{44}$. Data samples contained in the Human Protein Atlas clearly show that FGFR1 can localize to the nucleus (https://www.proteinatlas.org/ENSG00000077782-FGFR1). FGFR1 nuclear localization in three-dimensional model of breast cancer and pancreatic cancer can influence the expression of hundreds of genes and contribute to migratory phenotype ${ }^{45-47}$

(https://doi.org/10.1002/emmm.201302698). Additionally, in embryonic stem cells, FGFR1 nuclear localization may increase in developing brain cells during neuronal differentiation to Neuronal Progenitor Cells (NPC) ${ }^{46 ; 48 .}$

In this study, we investigated FGFR1 localization in HEK293 cells and two NB cell lines, SHSY5Y and SKNBE2, overexpressing both FGFR1 ${ }^{\text {wt }}$ and FGFR1 ${ }^{\text {N546K }}$ HEK293, SHSY5Y and SKNBE2 cells were transiently trasfected with pCMV6 expressing FGFR1 ${ }^{\text {wt }}$ or FGFR1 ${ }^{\text {N546K }}$ proteins and pCMV6 empty vector.

In HEK293, we examined FGFR1 ${ }^{\text {wt }}$ and FGFR1 ${ }^{\text {N546K }}$ proteins localization by ImageStream ${ }^{\mathrm{X}}$ Mark II Flow Cytometer (Fig. 3A). FGFR1 nuclear signal intensity

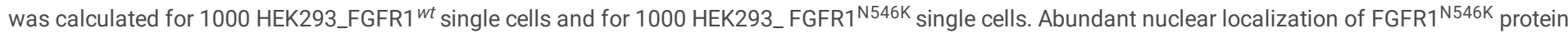
was statistically significant ( $\mathrm{p}$-value=0.0001). This observation was confirmed by immunofluorescence confocal microscopy assay showing FGFR 1 N546K protein mainly localized to nucleus, while FGFR $1^{\text {wt }}$ protein mainly localized to cytosol (Fig. 3B).

In SHSY5Y and SKNBE2 cell lines we observed a higher nuclear localization of the protein in FGFR1 ${ }^{\text {N546K }}$ overexpressing cells, compared to those overexpressing FGFR1 ${ }^{\text {wt }}$ (Fig. 3B).

These data were validated by western blot analysis on cytosol and nucleus fractions of HEK293, SHSY5Y and SKNBE2 trasfected cells (Fig. 3C).

\section{FGFR ${ }^{N 546 K}$ establishes crosstalk pathway activation and induces an increase in NB cellular invasion and colonigenicity}

Early studies reported FGFR1 ${ }^{\mathrm{N} 546 \mathrm{~K}}$ mutation affects the conformational dynamics of the tyrosine kinase domain, resulting in gain-of-function and ligandindependent constitutive activation 26;49;50.

We performed western blotting analysis on total protein extracts from SHSY5Y and SKNBE2 transiently transfected with pCMV6 expressing FGFR1 ${ }^{\text {wt }}$ or FGFR $1^{\text {N546K }}$ proteins and pCMV6 empty vector to evaluate phosphorylated and total FGFR1, STAT3, ERK and AKT levels. The t-GFP protein level was used as trasfection control and $\beta$-Actin was used as loading control.

In NB cell lines, FGFR1 ${ }^{\text {N546K }}$ overexpression enhanced the receptor kinase activity resulting in higher FGFR1 auto-phosphorylation. In addition, we observed a higher ERK, AKT and STAT3 phosphorylation in FGFR1 ${ }^{\text {N546K }}$ compared to FGFR1 ${ }^{\text {wt }}$ overexpressing cells (Fig. 4A).

We then evaluated cell viability in both SHSY5Y and SKNBE2 overexpressing FGFR $1^{\text {wt }}$ and FGFR $1^{\text {N546K }}$ protein compared to empty vector. Cell viability at $24 \mathrm{~h}$, $48 \mathrm{~h}$ and $72 \mathrm{~h}$ significantly increased in FGFR $1^{\text {wt }}$ and FGFR ${ }^{\mathrm{N} 546 \mathrm{~K}}$ overexpressing cells compared to pCMV6 empty vector ( $p$-value $\left.\leq 0.05\right)$ and FGFR ${ }^{\text {N5546K }}$ overexpressing cells had the highest cell viability ( $p$-value $\leq 0.05)$ (Fig. 4B).

The ability of transiently transfected SHSY5Y and SKNBE2 cells to invade and migrate through a matrigel-coated membrane support was evaluated. The number of invading FGFR ${ }^{w t}$ and FGFR1 ${ }^{\mathrm{N} 546 \mathrm{~K}}$ overexpressing cells increased significantly compared to control pCMV 6 cells. Interestingly, the number of invading FGFR1 ${ }^{\text {N546K }}$ overexpressing cells was even higher than the number of invading FGFR1 ${ }^{w t}$ overexpressing cells ( $p$-value $\left.\leq 0.05\right)($ Fig. $4 \mathrm{C}$ and $\mathrm{S} 2 \mathrm{C})$.

In addition, we analyzed the capability of FGFR1 ${ }^{w t}$ and FGFR1 ${ }^{\text {N546K }}$ overexpressing cells to interfere with colonigenicity in SHSY5Y and SKNBE2 cell lines. FGFR $1^{\text {wt }}$ overexpression resulted in an increase of colony number and colony area compared to the empty vector in both cell lines (Fig. 4D and S2D).

Moreover, FGFR1 ${ }^{\text {N546K }}$ overexpression was associated with the highest colony number and colony area in both cell lines (Fig. 4D and S2D).

N546K FGFR1 mutation may confer resistance to AZD4547 treatment in NB cell lines.

Since AZD4547 represents a small molecule inhibitor targeting FGFR1 aberrant activation currently used in clinical trial ${ }^{51 ; 52}$, we investigated the effects of this drug on FGFR1 ${ }^{\text {wt }}$ and FGFR1 ${ }^{\text {N546K }}$ in SHSY5Y and SKNBE2 cells.

Firstly, we evaluated AZD4547 potency against pCMV6-empty vector, FGFR $1^{\text {wt }}$ and FGFR1 ${ }^{\text {N546K }}$ overespressing cells (Fig. S3A). 
We performed cell viability assays in both cell lines testing different AZD4547 concentrations $(0.01 \mu \mathrm{M}, 0.1 \mu \mathrm{M}, 1 \mu \mathrm{M}$ and $10 \mu \mathrm{M})$, and then we calculated the half maximal inhibitory concentration (IC50) for this drug, which resulted comparable in SHSY5Y and SKNBE2 (Fig. S3A).

Based on the IC50 results, we selected the lower concentration of AZD4547 (0.1 $\mu \mathrm{M})$ able to decrease viability up to $20 \%$ in both cell lines (Fig. S3A). Specifically, we decided to not test $1 \mu \mathrm{M}$ AZD4547 because the treatment with this concentration showed a reduction of $31 \%$ of cell viability in SKNBE2 pCMV6-empty vector compared to vehicle cells (DMSO) (Fig. S3A).

To investigate the early effect of the drug treatment on the inhibition of downstream pathways, cells overexpressing FGFR $1^{\text {wt }}$ and FGFR ${ }^{\mathrm{N} 546 \mathrm{~K}}$ were incubated for 2 hours in serum-free medium in presence of AZD4547 $(0.1 \mu \mathrm{M})$ or vehicle (DMSO).

Total protein extracts were analyzed by western blotting and phosphorylation levels of FGFR1, STAT3, ERK and AKT were evaluated in relation to their respective total protein quotas. $\beta$-Actin protein levels were used as loading control (Fig. 5A, B).

In both cell lines overexpressing FGFR1 ${ }^{\text {wt }}$, AZD4547 0.1 $\mu \mathrm{M}$ decreased phospho-FGFR1, phospho-ERK and phospho-AKT protein levels, while did not strongly decrease phospho-STAT3 protein levels (Fig. 5A, B).

In SHSY5Y overexpressing FGFR1 ${ }^{\text {N546K }}$, AZD4547 did not show efficacy to decrease phospho-FGFR1, phospho-ERK, phospho-AKT and phospho-STAT3 protein levels, that remained aboundant in cells (Fig. 5A). In SKNBE2 overexpressing FGFR1 ${ }^{\text {N546K }}$, although AZD4547 0.1 $\mu$ M decreased phospho-FGFR1 and phospho-ERK levels, phospho-AKT levels were not affected and phospho- STAT3 levels resulted even enhanced (Fig. 5B).

In line with western blotting results (Fig. 5A, B), AZD4547 0.1 $\mathrm{MM}$ treatment, by impairing FGFR1 signaling, led to a reduction by almost 50\% of invasive capacity (Fig. 5C and S4A) and colony number (Fig. 5D and S4B) in both SHSY5Y and SKNBE2 FGFR1 ${ }^{\text {wt }}$ overexpressing cells compared to untreated cells.

In SHSY5Y FGFR1 ${ }^{\text {N546K }}$ overexpressing cells AZD4547 0.1 $\mu \mathrm{M}$ treatment, that increased phospho-ERK levels and unaffected phospho-FGFR1 and phosphoAKT levels as previously shown (Fig. 5A), did not strongly impair cellular invasion (Fig. 5C and S4A) and neurospheres formation capability (Fig. 5D and S4B). On the other hand, we observed an increase in cellular invasion capacity (Fig. 5C and S4A) and in colony number (Fig. 5D and S4B) in SKNBE2 FGFR1 N546K overexpressing cells, probably due to STAT3 and AKT phosphorylation (Fig. 5B).

Altogheter, these data suggest that AZD4547 abolishes the pathway activation induced by FGFR1 ${ }^{\text {wt }}$, but does not show a great effectiveness on those ehanced by FGFR1 ${ }^{\text {N546K }}$. Hence, N546K mutation may establish a resistance to AZD4547 treatment through activation of AKT and STAT3 pathways.

\section{Targeting of FGFR1 ${ }^{\text {N546K }}$ signaling by combination treatment with AZD4547 and GDC0941 decreases crosstalk pathways activation, invasion and neurosphere formation capability.}

Since AZD4547 alone resulted non-effective in the abolishment of FGFR1 ${ }^{\text {N546K }}$ induced cross-pathways, we choosed to use it in combination with GDC0941, a $\mathrm{PI3K}$ inhibitor already used in clinical trials ${ }^{53 ; 54}$.

As previously done for AZD4547, we firstly tested different concentrations of GDC0941 (0.01 $\mu \mathrm{M}, 0.1 \mu \mathrm{M}, 1 \mu \mathrm{M}$ and $10 \mu \mathrm{M})$ alone in both cell lines transiently transfected with FGFR $1^{\text {wt }}$ and FGFR ${ }^{\mathrm{N} 456 \mathrm{~K}}$ by performing cell viability assay (Fig. S3B). Differently from AZD3547, GDC0941 IC50 was higher in SKNBE2 cells (Fig. S3A, B).

Based on the IC50 results, we choosed to test the combination of AZD4547 $(0.1 \mu \mathrm{M})$ and GDC0941 $(0.1 \mu \mathrm{M}$ and $1 \mu \mathrm{M})$ on cell viability, and we selected the lower concentrations able to decrease viability up to $20 \%$ (Fig. S3C). Particularly, we used two GDC0941 concentrations $(0.1 \mu \mathrm{M}$ and $1 \mu \mathrm{M})$ since GDC0941 has shown lower toxicity in SKNBE2 (Fig. S3B, C).

To investigate the early effects of the combination treatment on the inhibition of downstream pathways, cells overexpressing FGFR $1^{\text {wt }}$ and FGFR $1^{\text {N546K }}$ were incubated for 2 hours in serum-free medium in presence of AZD4547 $(0.1 \mu \mathrm{M})$ and GDC0941 (0.1 $\mu \mathrm{M}$ and $1 \mu \mathrm{M})$ or vehicle (DMSO).

Our aim was to investigate if these combinations at low doses could be more effective than AZD4547 single treatment in NB cells overespressing FGFR1 ${ }^{\text {N546K. }}$.

The transfected cells were treated with single GDC0941 $(0.1 \mu \mathrm{M}$ or $1 \mu \mathrm{M})$ to test drug efficiency. In FGFR1 ${ }^{\mathrm{N} 546 \mathrm{~K}}$ overexpressing cells treated with GDC0941 alone, we observed a significant decrease only in phospho-AKT protein levels (Figure 5A, B).

In cells overexpressing FGFR $1^{\text {wt }}$, the combination treatment with AZD4547 $(0.1 \mu \mathrm{M})$ and GDC0941 $(0.1 \mu \mathrm{M}$ or $1 \mu \mathrm{M})$ was not effective to decrease both phospho-STAT3 and phospho-ERK protein levels, which in contrast showed an increase probably due to a compensation mechanism following the inhibition of FGFR1 signaling (Figure 5A, B). Of note, in both cell lines overexpressing FGFR1 ${ }^{\text {N546K }}$, the combination of AZD4547 0.1 $\mu$ M and GDC0941 $1 \mu$ M showed the best in vitro efficacy for the inhibition of all the three examinated pathways, highlighted by the reduction of phosphorylated/total protein levels (Figure $5 \mathrm{~A}$, B).

In SHSY5Y cells overexpressing FGFR1 ${ }^{\text {wt }}$ protein, AZD4547 $0.1 \mu \mathrm{M}$ and GDC0941 1 $\mu \mathrm{M}$ combination, compared to AZD4547 single treatment, showed a lower reduction in cell invasion capability (Fig. 5C and S4A) and a decrease of colony number higher than 50\% (Fig. 5D and S4B), probably due to increment of phosoho-STAT3 and a strong decrease of phoshpo-AKT levels, respectively (Fig. 5A). In SKNBE2 overexpressing FGFR1 ${ }^{\text {wt }}$, the combination and AZD4547 single treatment showed a similar effect on cell invasion (Fig. 5C and S4A) and colonigenic (Fig. 5D and S4B) capacity, as result of similar downstream

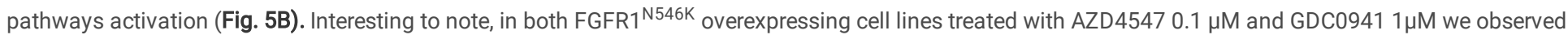


a reduction of over $50 \%$ of invasion and neurosphere capacity, as consequence of downstream pathway impairments aboved mentioned (Fig. 5A-C and S4A, B).

Together, these results highlight that AZD4547 $0.1 \mu \mathrm{M}$ and GDC0941 $1 \mu \mathrm{M}$ combination treatment was able to decrease the activation of downstream



Therefore, AZD4547 and GDC0941 combination treatment may represent a promising therapeutic strategy to overcome the resistance mechanisms induced by FGFR1 N546K mutation under AZD4547 treatment alone.

\section{Discussion And Conclusion}

FGFR1 is an emerging promising target for the treatment of adult cancers as breast, lung and gastric cancers with FGFR1 amplification being the most common somatic alteration responsive to therapeutic intervention. However, although we found no FGFR1 amplifications in NB samples, point mutations seemed to occur in primary and relapse tumors ${ }^{16 ; 18 ; 25}$. Here we re-analyzed the DNA coding and noncoding sequences of FGFR1 gene in 19 matched primary and relapsed NB tumors and found the hotspot mutation N546K in one sample at diagnosis and relapse obtained by TARGET database repository, suggesting that this mutation undergoes clonal selection. Of note, a large sequencing study recently found the same mutation in 6 primary NB tumors and in one matched relapsed tumor sample ${ }^{25}$. FGFR1 clone selection for rare resistant subclones has been also reported in lung and colorectal resistant tumors, thus revealing a change in variant allele frequency of FGFR1 somatic variants ${ }^{55 ; 56}$. Of note, N546K mutation was also found in Ewing sarcoma and brain tumors 27-31.

Cancer process is thought to be triggered by the reactivation of embryonic mechanisms in stem cells of adult tissues, in an entirely inappropriate context ${ }^{57}$. In line with this observation, we have recently shown that altered expression of genes involved in embryogenesis, due to cancer risk genetic variants, may contribute to malignancy and metastasis in neural crest-derived tumors including NB ${ }^{58}$.

FGFR1 activation in resistent- or advanced-tumors is consistent with an epithelial-to-mesenchymal transition (EMT) and FGFR1 nuclear localization ${ }^{59-62}$. Particularly, FGFR1 nuclear form is crucial for the expression of embryonic stem cell migration and neural crest formation genes and promotes also invasion and extracellular matrix changes in advanced pancreatic and breast cancer cells ${ }^{62 ; 63}$. Considering these previous findings, we hypothesize that FGFR1 can act as a cancer-driver gene in NB and that mutations in this gene might activate embryonic signaling, therefore promoting the recurrence of the disease. Our in vitro data show that FGFR1 silencing in NB cells impairs cell proliferation, cell invasion and cell growth and these effects are rescued in FGFR1 ${ }^{\text {wt }}$ overexpressing cells. Accordingly, our gene expression analysis of different datasets showed that high FGFR1 associated with metastatic and relapsed tumors and inferior relapse free survival, suggesting its role in promoting disease progression and recurrence. Our data show that N546K mutation leads to nuclear localization of FGFR1 protein and to activation of downstream signaling (AKT and STAT3) which results in an increase of the invasive and colonigenic capacity of cells. Since FGFR1 can promote the activation of developmental genes in embryonic stem cells (ESCs) ${ }^{64}$, we do not exclude that N546K may lead to a reactivation of embryonic signaling as a result of FGFR1 nuclear localization.

FGFR1 is a tyrosine kinase receptor that, once activated, phosphorylates specialized intracellular adapters upstream of MAPK1/2 signaling pathway and its inhibitors are broadly used in clinical trials for the treatment of breast, lung and gastric cancers with FGFR1 amplification 51;52;65. AZD4547 is a small molecule tyrosine kinase inhibitor (TKI) able to inactivate FGFRs downstream signaling by occupying the ATP-binding pocket in the kinase domain ${ }^{66}$. It has been reported as one of the most effective compounds for FGFR1 signaling inhibition that can be used at low concentrations for the treatment of advanced tumors ${ }^{67-69}$. In FGFR1 ${ }^{\text {wt }}$ overexpressing cells, we observed that AZD4547 treatment was sufficient to abrogate FGFR1 signaling throught inhibition of phospho-FGFR1 and phospho-ERK activation, resulting in an impairement of invasion and colonigenic cell ability. On the other hand, in FGFR1 ${ }^{\text {N546K }}$ overexpressing cells, treatment with AZD4547 alone lead to an increase of phospho-AKT and phospho-STAT3 levels. These findings further support that AZD4547 treatment by targeting FGFR1 can induce resistance mechanisms ${ }^{70-75}$.

Mostly potential resistance mechanisms to FGFR1 inhibition can converge on de novo ${ }^{76 ; 77}$ and/or re-activation ${ }^{78}$ of several signaling cascades. In particular, the mechanisms of AZD4547 resistance involve gene fusion (JUDMID-BRAF), alternative pathways activation (RAS-MAPK, ErbB3/PI3K/AKT and MET pathways) and related molecular abnormalities (RASA1, PHLDA1, PTEN, STAT3) ${ }^{70 ; 75}$. As additional mutations or selection of clones present prior to treatment might activate resistance mechanisms, we hypothesize that therapeutic combination of FGFR1 and PI3K inhibitors may have a synergistic effect respect to FGFR1 inhibitor used alone. Several studies have shown that GDC0941, designed to bind the ATP-binding pocket of PI3K to prevent formation of phosphatidylinositol-3, 4, 5- triphosphate (PIP3), inhibits cell proliferation in vitro and in vivo ${ }^{79 ; 80}$. GDC0941 molecule is already used in clinical trials in combination with other drugs for the treatment of metastatic breast cancers ${ }^{53 ; 54}$. Here, we observed that the combination of AZD4547 and GDC0941 shows additive effect on malignant phenotypes in vitro by inhibiting STAT3, AKT and ERK signaling activated by FGFR1 ${ }^{\text {N564K }}$ protein.

Taken together, our results suggest that FGFR1 expression is crucial for NB progression. Preliminary findings further suggest that FGFR1N546K overexpressing cells show a further increase in motility and a failure to respond to treatment with FGFR1 inhibitor by activating ERK, STAT3 and AKT pathways. These signaling cascades enhanced by N546K mutation can be suppressed using the combination of FGFR1 and its downstream pathways inhibitors.

Therefore, targeting FGFR1 mutation may represent a promising clinical strategy for both preventing and overcoming acquired drug resistance and provide insights regarding potential precision medicine therapeutics to achieve the complete remission in high-risk NB.

\section{Declarations}




\section{Ethical Approval and Consent to participate}

Ethics committee of Ospedale Pediatrico “Bambino Gesù” approved this study (Protocol: 20757). Informed consent was obtained from all individual participants included in the study.

\section{Consent for publication}

All authors consent to the publication of the manuscript in Cancer Cell International.

\section{Availability of data and materials}

The datasets generated during and/or analyzed during the current study are available from the corresponding author on reasonable request.

\section{Competing interests}

Authors declare no conflict of interests.

\section{Funding}

This study was supported by grants from Associazione Italiana per la Ricerca sul Cancro (Grant no. 25796 to M.C. and Grant no. 20757 to A.l. and Special Project 5×1000 no. 9962 to F.L.), Fondazione Italiana per la Lotta al Neuroblastoma (to M.C.); Associazione Oncologia Pediatrica e Neuroblastoma (to M.C.) and Fondazione Umberto Veronesi (to F.C.) and Regione Campania "SATIN" grant 2018-2020 (to MC)

\section{Author contributions}

FC and AM designed research, performed the experiments, interpreted the data and drafted the manuscript. MT, MA, FV, SC and TM performed the experiments. VAL performed bioinformatic analysis and interpreted the data. AC, BDA, MM contributed with patient samples and clinical information. MC, AI and FL formulated the strategy and supervised the research.

All authors read and approved the final version of the work to be published.

\section{Acknowledgements}

The authors want to thank the the BIT_Gaslini Biobank, IRCCS Istituto Giannina Gaslini, Via G. Gaslini, 5, Genova, Italy, for providing us with specimens.

\section{References}

1. Matthay KK, Maris JM, Schleiermacher G, et al. Neuroblastoma. Nat Rev Dis Primers. 2016;2:16078.

2. Capasso M, Diskin SJ. Genetics and genomics of neuroblastoma. Cancer Treat Res. 2010;155:65-84.

3. Lasorsa VA, Cimmino F, Ognibene M, et al. 19p loss is significantly enriched in older age neuroblastoma patients and correlates with poor prognosis. NPJ Genom Med. 2020;5:18.

4. Capasso M, Diskin SJ, Totaro F, et al. Replication of GWAS-identified neuroblastoma risk loci strengthens the role of BARD1 and affirms the cumulative effect of genetic variations on disease susceptibility. Carcinogenesis. 2013;34(3):605-611.

5. McDaniel LD, Conkrite KL, Chang X, et al. Common variants upstream of MLF1 at 3q25 and within CPZ at 4p16 associated with neuroblastoma. PLoS Genet. 2017;13(5):e1006787.

6. Avitabile M, Succoio M, Testori A, et al. Neural crest-derived tumor neuroblastoma and melanoma share $1 \mathrm{p} 13.2$ as susceptibility locus that shows a longrange interaction with the SLC16A1 gene. Carcinogenesis. 2019.

7. Cimmino F, Avitabile M, Diskin SJ, et al. Fine mapping of $2 q 35$ high-risk neuroblastoma locus reveals independent functional risk variants and suggests full-length BARD1 as tumor-suppressor. Int J Cancer. 2018;143(11):2828-2837.

8. Capasso M, McDaniel LD, Cimmino F, et al. The functional variant rs34330 of CDKN1B is associated with risk of neuroblastoma. J Cell Mol Med. 2017;21(12):3224-3230.

9. Capasso M, Montella A, Tirelli M, Maiorino T, Cantalupo S, Iolascon A. Genetic Predisposition to Solid Pediatric Cancers. Front Oncol. 2020;10:590033.

10. Tonini GP, Capasso M. Genetic predisposition and chromosome instability in neuroblastoma. Cancer Metastasis Rev. 2020;39(1):275-285.

11. Luksch R, Castellani MR, Collini P, et al. Neuroblastoma (Peripheral neuroblastic tumours). Crit Rev Oncol Hematol. 2016;107:163-181.

12. Russo R, Cimmino F, Pezone L, et al. Kinome expression profiling of human neuroblastoma tumors identifies potential drug targets for ultra high-risk patients. Carcinogenesis. 2017;38(10):1011-1020.

13. Formicola D, Petrosino G, Lasorsa VA, et al. An 18 gene expression-based score classifier predicts the clinical outcome in stage 4 neuroblastoma. $J$ Trans/ Med. 2016;14(1):142.

14. Barbieri E, De Preter K, Capasso M, et al. A p53 drug response signature identifies prognostic genes in high-risk neuroblastoma. PLoS One. 2013;8(11):e79843.

15. Eleveld TF, Oldridge DA, Bernard V, et al. Relapsed neuroblastomas show frequent RAS-MAPK pathway mutations. Nat Genet. $2015 ; 47(8): 864-871$. 
16. Padovan-Merhar OM, Raman P, Ostrovnaya I, et al. Enrichment of Targetable Mutations in the Relapsed Neuroblastoma Genome. PLoS Genet. 2016;12(12):e1006501.

17. Schramm A, Koster J, Assenov Y, et al. Mutational dynamics between primary and relapse neuroblastomas. Nat Genet. 2015;47(8):872-877.

18. Lasorsa VA, Formicola D, Pignataro P, et al. Exome and deep sequencing of clinically aggressive neuroblastoma reveal somatic mutations that affect key pathways involved in cancer progression. Oncotarget. 2016;7(16):21840-21852.

19. Esposito MR, Binatti A, Pantile M, et al. Somatic mutations in specific and connected subpathways are associated with short neuroblastoma patients' survival and indicate proteins targetable at onset of disease. Int J Cancer. 2018;143(10):2525-2536.

20. Peifer M, Hertwig F, Roels F, et al. Telomerase activation by genomic rearrangements in high-risk neuroblastoma. Nature. 2015;526(7575):700-704.

21. Andolfo I, Lasorsa VA, Manna F, et al. Kinome multigenic panel identified novel druggable EPHB4-V871I somatic variant in high-risk neuroblastoma. $J$ Cell Mol Med. 2020;24(11):6459-6471.

22. Deveau P, Colmet Daage L, Oldridge D, et al. QuantumClone: clonal assessment of functional mutations in cancer based on a genotype-aware method for clonal reconstruction. Bioinformatics. 2018;34(11):1808-1816.

23. Capasso M, Lasorsa VA, Cimmino F, et al. Transcription factors involved in tumorigenesis are over-represented in mutated active DNA binding sites in neuroblastoma. Cancer Res. 2019.

24. Consortium APG. AACR Project GENIE: Powering Precision Medicine through an International Consortium. Cancer Discov. 2017;7(8):818-831.

25. Brady SW, Liu Y, Ma X, et al. Pan-neuroblastoma analysis reveals age- and signature-associated driver alterations. Nat Commun. 2020;11(1):5183.

26. Lew ED, Furdui CM, Anderson KS, Schlessinger J. The precise sequence of FGF receptor autophosphorylation is kinetically driven and is disrupted by oncogenic mutations. Sci Signal. 2009;2(58):ra6.

27. Jones DT, Hutter B, Jager N, et al. Recurrent somatic alterations of FGFR1 and NTRK2 in pilocytic astrocytoma. Nat Genet. 2013;45(8):927-932.

28. Ng PK, Li J, Jeong KJ, et al. Systematic Functional Annotation of Somatic Mutations in Cancer. Cancer Cell. 2018;33(3):450-462 e410.

29. Agelopoulos K, Richter GH, Schmidt E, et al. Deep Sequencing in Conjunction with Expression and Functional Analyses Reveals Activation of FGFR1 in Ewing Sarcoma. Clin Cancer Res. 2015;21(21):4935-4946.

30. Kordacka J, Zakrzewski K, Gruszka R, et al. Sensitive detection of FGFR1 N546K mosaic mutation in patient with encephalocraniocutaneous lipomatosis and pilocytic astrocytoma. Am J Med Genet A. 2019;179(8):1622-1627.

31. Appay R, Fina F, Barets D, et al. Multiplexed Droplet Digital PCR Assays for the Simultaneous Screening of Major Genetic Alterations in Tumors of the Central Nervous System. Front Oncol. 2020;10:579762.

32. Porta R, Borea R, Coelho A, et al. FGFR a promising druggable target in cancer: Molecular biology and new drugs. Crit Rev Oncol Hematol. 2017;113:256267.

33. Cibulskis K, Lawrence MS, Carter SL, et al. Sensitive detection of somatic point mutations in impure and heterogeneous cancer samples. Nat Biotechnol. 2013;31(3):213-219.

34. Saunders CT, Wong WS, Swamy S, Becq J, Murray LJ, Cheetham RK. Strelka: accurate somatic small-variant calling from sequenced tumor-normal sample pairs. Bioinformatics. 2012;28(14):1811-1817.

35. Pugh TJ, Morozova O, Attiyeh EF, et al. The genetic landscape of high-risk neuroblastoma. Nat Genet. 2013;45(3):279-284.

36. Wang K, Li M, Hakonarson H. ANNOVAR: functional annotation of genetic variants from high-throughput sequencing data. Nucleic Acids Res. 2010;38(16):e164.

37. Fu Y, Liu Z, Lou S, et al. FunSeq2: a framework for prioritizing noncoding regulatory variants in cancer. Genome Biol. 2014;15(10):480.

38. Attiyeh EF, Diskin SJ, Attiyeh MA, et al. Genomic copy number determination in cancer cells from single nucleotide polymorphism microarrays based on quantitative genotyping corrected for aneuploidy. Genome Res. 2009;19(2):276-283.

39. Nilsen G, Liestol K, Van Loo P, et al. Copynumber: Efficient algorithms for single- and multi-track copy number segmentation. BMC Genomics. 2012;13:591.

40. Cimmino F, Pezone L, Avitabile M, et al. Inhibition of hypoxia inducible factors combined with all-trans retinoic acid treatment enhances glial transdifferentiation of neuroblastoma cells. Sci Rep. 2015;5:11158.

41. Cimmino F, Avitabile M, Lasorsa VA, et al. Functional characterization of full-length BARD1 strengthens its role as a tumor suppressor in neuroblastoma. $J$ Cancer. 2020;11(6):1495-1504.

42. Cimmino F, Spano D, Capasso M, et al. Comparative proteomic expression profile in all-trans retinoic acid differentiated neuroblastoma cell line. $J$ Proteome Res. 2007;6(7):2550-2564.

43. Cimmino F, Avitabile M, Pezone L, et al. CD55 is a HIF-2alpha marker with anti-adhesive and pro-invading properties in neuroblastoma. Oncogenesis. 2016;5:e212.

44. Stachowiak EK, Maher PA, Tucholski J, et al. Nuclear accumulation of fibroblast growth factor receptors in human glial cells-association with cell proliferation. Oncogene. 1997;14(18):2201-2211.

45. Wendt MK, Taylor MA, Schiemann BJ, Sossey-Alaoui K, Schiemann WP. Fibroblast growth factor receptor splice variants are stable markers of oncogenic transforming growth factor beta1 signaling in metastatic breast cancers. Breast Cancer Res. 2014;16(2):R24.

46. Stachowiak MK, Stachowiak EK. Evidence-Based Theory for Integrated Genome Regulation of Ontogeny-An Unprecedented Role of Nuclear FGFR1 Signaling. J Cell Physiol. 2016;231(6):1199-1218. 
47. Infante JR, Camidge DR, Mileshkin LR, et al. Safety, pharmacokinetic, and pharmacodynamic phase I dose-escalation trial of PF-00562271, an inhibitor of focal adhesion kinase, in advanced solid tumors. J Clin Oncol. 2012;30(13):1527-1533.

48. Decker B, Liput M, Abdellatif H, et al. Global Genome Conformational Programming during Neuronal Development Is Associated with CTCF and Nuclear FGFR1-The Genome Archipelago Model. Int J Mol Sci. 2020;22(1).

49. Chen H, Ma J, Li W, et al. A molecular brake in the kinase hinge region regulates the activity of receptor tyrosine kinases. Mol Cell. 2007;27(5):717-730.

50. Rand V, Huang J, Stockwell T, et al. Sequence survey of receptor tyrosine kinases reveals mutations in glioblastomas. Proc Natl Acad Sci U S A. 2005;102(40):14344-14349.

51. Chae YK, Hong F, Vaklavas C, et al. Phase II Study of AZD4547 in Patients With Tumors Harboring Aberrations in the FGFR Pathway: Results From the NCI-MATCH Trial (EAY131) Subprotocol W. J Clin Oncol. 2020;38(21):2407-2417.

52. Paik PK, Shen R, Berger MF, et al. A Phase lb Open-Label Multicenter Study of AZD4547 in Patients with Advanced Squamous Cell Lung Cancers. Clin Cancer Res. 2017;23(18):5366-5373.

53. Vuylsteke P, Huizing M, Petrakova K, et al. Pictilisib PI3Kinase inhibitor (a phosphatidylinositol 3-kinase [PI3K] inhibitor) plus paclitaxel for the treatment of hormone receptor-positive, HER2-negative, locally recurrent, or metastatic breast cancer: interim analysis of the multicentre, placebo-controlled, phase II randomised PEGGY study. Ann Oncol. 2016;27(11):2059-2066.

54. Rimawi M, Ferrero JM, de la Haba-Rodriguez J, et al. First-Line Trastuzumab Plus an Aromatase Inhibitor, With or Without Pertuzumab, in Human Epidermal Growth Factor Receptor 2-Positive and Hormone Receptor-Positive Metastatic or Locally Advanced Breast Cancer (PERTAIN): A Randomized, Open-Label Phase II Trial. J Clin Oncol. 2018;36(28):2826-2835.

55. Bray SM, Lee J, Kim ST, et al. Genomic characterization of intrinsic and acquired resistance to cetuximab in colorectal cancer patients. Sci Rep. 2019;9(1):15365.

56. Raoof S, Mulford IJ, Frisco-Cabanos H, et al. Targeting FGFR overcomes EMT-mediated resistance in EGFR mutant non-small cell lung cancer. Oncogene. 2019;38(37):6399-6413.

57. Cofre J, Abdelhay E. Cancer Is to Embryology as Mutation Is to Genetics: Hypothesis of the Cancer as Embryological Phenomenon. ScientificWorldJournal. 2017;2017:3578090.

58. Avitabile M, Succoio M, Testori A, et al. Neural crest-derived tumor neuroblastoma and melanoma share $1 \mathrm{p} 13.2$ as susceptibility locus that shows a longrange interaction with the SLC16A1 gene. Carcinogenesis. 2020;41(3):284-295.

59. Acevedo VD, Gangula RD, Freeman KW, et al. Inducible FGFR-1 activation leads to irreversible prostate adenocarcinoma and an epithelial-to-mesenchymal transition. Cancer Cell. 2007;12(6):559-571.

60. Wang K, Ji W, Yu Y, et al. Correction: FGFR1-ERK1/2-SOX2 axis promotes cell proliferation, epithelial-mesenchymal transition, and metastasis in FGFR1amplified lung cancer. Oncogene. 2020;39(42):6619-6620.

61. Servetto A, Kollipara R, Formisano L, et al. Nuclear FGFR1 Regulates Gene Transcription and Promotes Antiestrogen Resistance in ER(+) Breast Cancer. Clin Cancer Res. 2021;27(15):4379-4396.

62. Chioni AM, Grose R. FGFR1 cleavage and nuclear translocation regulates breast cancer cell behavior. J Cell Biol. 2012;197(6):801-817.

63. Coleman SJ, Chioni AM, Ghallab M, et al. Nuclear translocation of FGFR1 and FGF2 in pancreatic stellate cells facilitates pancreatic cancer cell invasion. EMBO Mol Med. 2014;6(4):467-481.

64. Terranova C, Narla ST, Lee YW, et al. Global Developmental Gene Programing Involves a Nuclear Form of Fibroblast Growth Factor Receptor-1 (FGFR1). PLoS One. 2015;10(4):e0123380.

65. Van Cutsem E, Bang YJ, Mansoor W, et al. A randomized, open-label study of the efficacy and safety of AZD4547 monotherapy versus paclitaxel for the treatment of advanced gastric adenocarcinoma with FGFR2 polysomy or gene amplification. Ann Oncol. 2017;28(6):1316-1324

66. Katoh M. FGFR inhibitors: Effects on cancer cells, tumor microenvironment and whole-body homeostasis (Review). Int J Mol Med. 2016;38(1):3-15.

67. Cheng W, Wang M, Tian X, Zhang X. An overview of the binding models of FGFR tyrosine kinases in complex with small molecule inhibitors. Eur J Med Chem. 2017;126:476-490.

68. Wan X, Corn PG, Yang J, et al. Prostate cancer cell-stromal cell crosstalk via FGFR1 mediates antitumor activity of dovitinib in bone metastases. Sci Trans/ Med. 2014;6(252):252ra122.

69. Turner N, Grose R. Fibroblast growth factor signalling: from development to cancer. Nat Rev Cancer. 2010;10(2):116-129.

70. Zhou Y, Wu C, Lu G, Hu Z, Chen Q, Du X. FGF/FGFR signaling pathway involved resistance in various cancer types. J Cancer. 2020;11(8):2000-2007.

71. Ryan MR, Sohl CD, Luo B, Anderson KS. The FGFR1 V561M Gatekeeper Mutation Drives AZD4547 Resistance through STAT3 Activation and EMT. Mol Cancer Res. 2019;17(2):532-543.

72. Phanhthilath N, Hakim S, Su C, et al. Mechanisms of Efficacy of the FGFR1-3 Inhibitor AZD4547 in Pediatric Solid Tumor Models. Invest New Drugs. 2020;38(6):1677-1686.

73. Wang L, Sustic T, Leite de Oliveira R, et al. A Functional Genetic Screen Identifies the Phosphoinositide 3-kinase Pathway as a Determinant of Resistance to Fibroblast Growth Factor Receptor Inhibitors in FGFR Mutant Urothelial Cell Carcinoma. Eur Urol. 2017;71(6):858-862.

74. Kas SM, de Ruiter JR, Schipper K, et al. Transcriptomics and Transposon Mutagenesis Identify Multiple Mechanisms of Resistance to the FGFR Inhibitor AZD4547. Cancer Res. 2018;78(19):5668-5679.

75. Yue S, Li Y, Chen X, et al. FGFR-TKI resistance in cancer: current status and perspectives. J Hematol Oncol. 2021;14(1):23. 
76. Gimenez-Xavier P, Pros E, Aza A, et al. Deep analysis of acquired resistance to FGFR1 inhibitor identifies MET and AKT activation and an expansion of AKT1 mutant cells. Oncotarget. 2018;9(59):31549-31558.

77. Datta J, Damodaran S, Parks H, et al. Akt Activation Mediates Acquired Resistance to Fibroblast Growth Factor Receptor Inhibitor BGJ398. Mol Cancer Ther. 2017;16(4):614-624.

78. Luo H, Quan J, Xiao H, et al. FGFR inhibitor AZD4547 can enhance sensitivity of esophageal squamous cell carcinoma cells with epithelialmesenchymal transition to gefitinib. Oncol Rep. 2018;39(5):2270-2278.

79. Folkes AJ, Ahmadi K, Alderton WK, et al. The identification of 2-(1H-indazol-4-yl)-6-(4-methanesulfonyl-piperazin-1-ylmethyl)-4-morpholin-4-yl-t hieno[3,2d]pyrimidine (GDC-0941) as a potent, selective, orally bioavailable inhibitor of class I PI3 kinase for the treatment of cancer. J Med Chem. 2008;51(18):5522-5532.

80. Knight ZA, Shokat KM. Chemically targeting the PI3K family. Biochem Soc Trans. 2007;35(Pt 2):245-249.

\section{Figures}

A
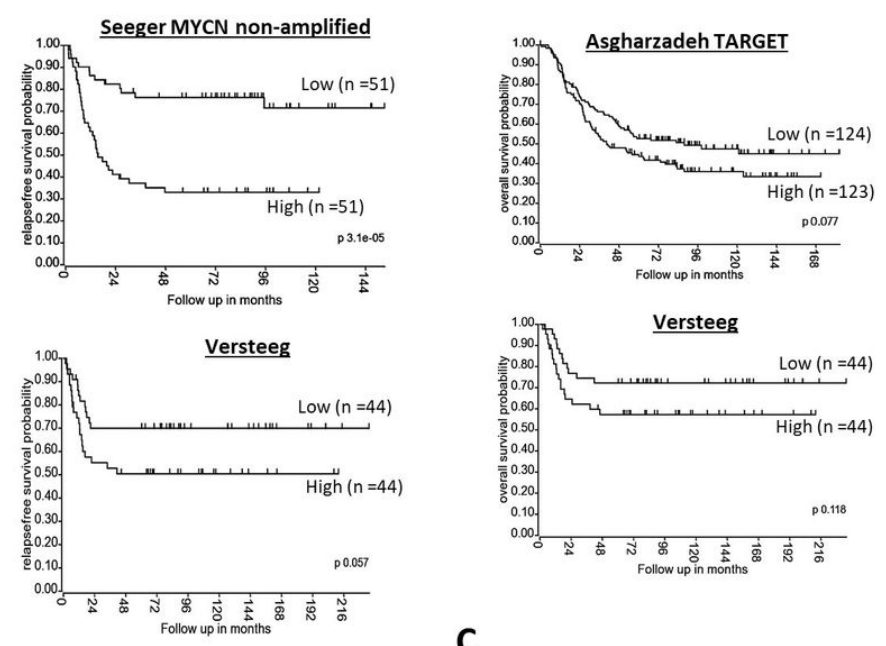

B

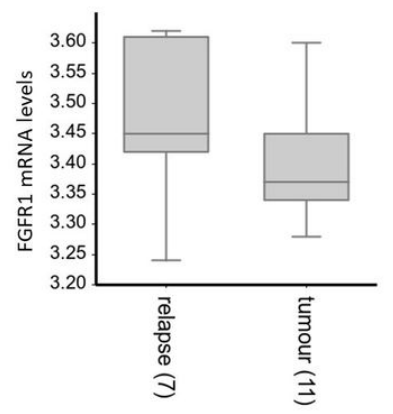

C

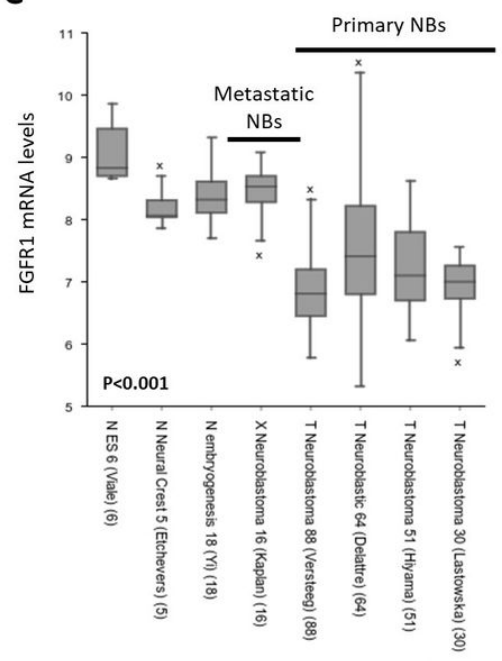

Figure 1

Association of FGFR1 expression with clinical outcomes in patients with NB. (A) The association of FGFR1 expression with clinical outcomes was evaluated in the following datasets: Seeger, Asgharzadeh TARGET and Versteeg (http://hgserver1.amc.nl/cgi-bin/r2/main.cgi). ( $n$ =number of patients). (B) FGFR1 expression analysis in datasets of primary and relapsed tumors. (C) FGFR1 expression levels in two embryonic cells (ES), one neuronal crest cells (NC), one metastatic xenograft tumors $(X)$ and four primary NB $(T)$ datasets. In $(B)$ and $(C)$ the number of samples is reported in brackets. $p=p$-value 
A
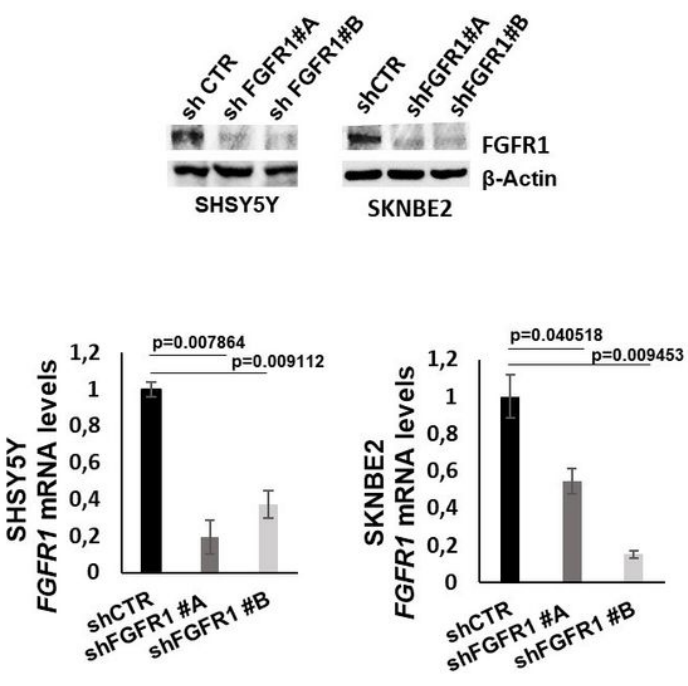

C


B
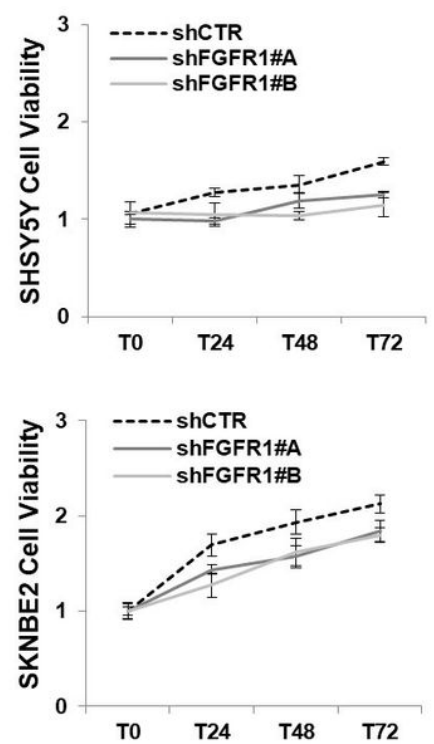

D


\section{Figure 2}

FGFR1 silencing impairs cell growth, cell invasion and clonogenicity in NB cells. (A) FGFR1 silencing efficiency was evaluated by western blotting and RT-PCR, in SHSY5Y and SKNBE2 transduced by lentiviral vectors encoding shFGFR1\#A and shFGFR1\#B. FGFR1 folded mRNA levels are reported. (B) Cell viability in shFGFR1\#A and shFGFR1\#B cells is shown as fold change respect to the control (shCTR). (C) Invading cells and (D) colony number in FGFR1 silenced and control cells are reported. Vehicle $=$ DMSO. $p=p$-value 
A

HEK293_FGFR1 wt

HEK293_FGFR1 N546K

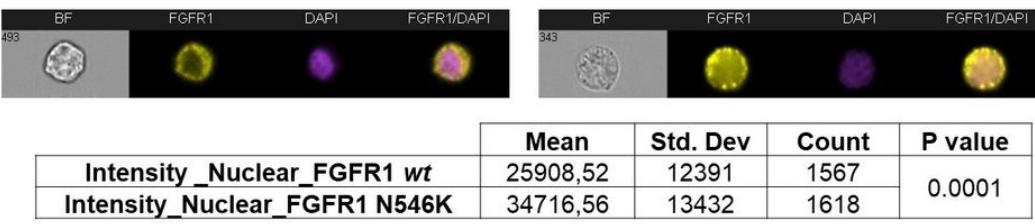

\section{B}
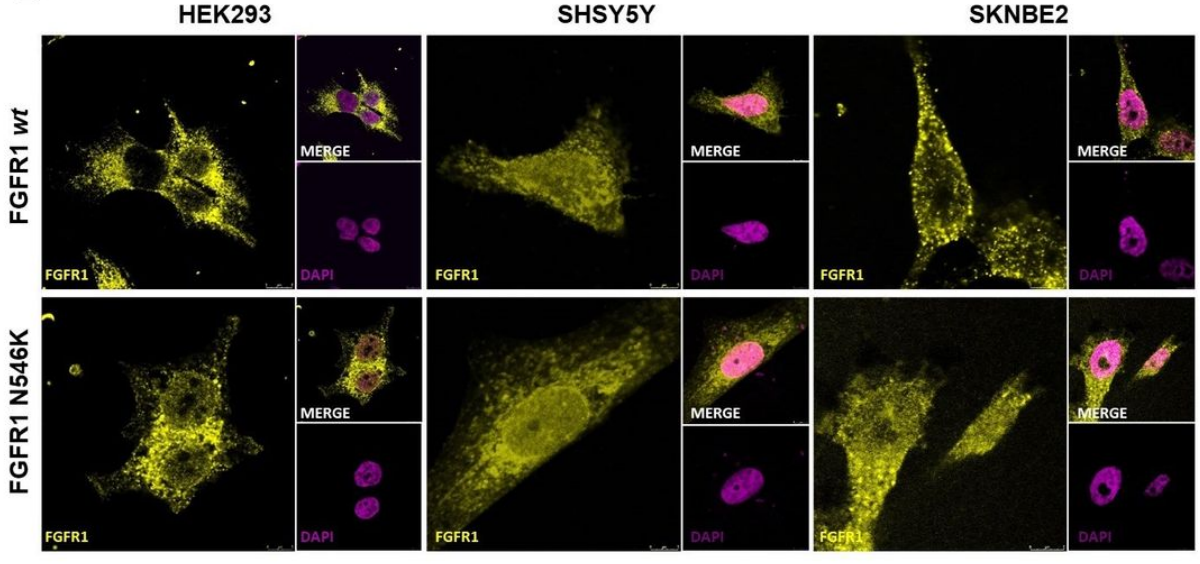

C
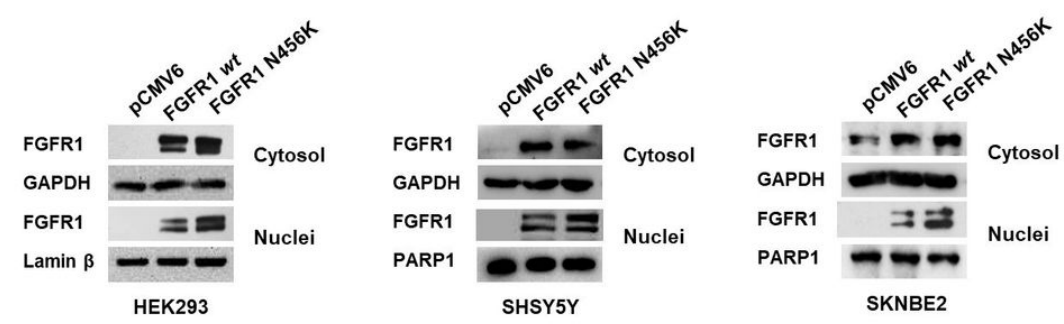

Figure 3

FGFR $1^{\text {wt }}$ and FGFR $1^{\text {N546K }}$ proteins localization. (A) FGFR1 localization in HEK293 overxspressing FGFR $1^{\text {wt }}$ or FGFR $^{\text {N546K }}$ protein was analyzed by Image Stream flow Citometry. Above are shown the representative figures of single cells at $60 x$ magnification, in the table are reported the values of mean intensity, standard deviation, cells counted and $p$-value. (B) Immunostaining of HEK293, SHSY5Y and SKNBE2 transfected with FGFR ${ }^{\text {wt }}$ or FGFR1 ${ }^{\text {N546K }}$ analyzed by confocal microscopy. (C) FGFR1 protein levels in both cytosol and nucleus protein fractions from HEK293, SHSY5Y and SKNBE2 trasfected cells was evaluated by western blotting. GAPDH, Lamin $\beta$ and PARP1 protein levels were used as loading controls 
A

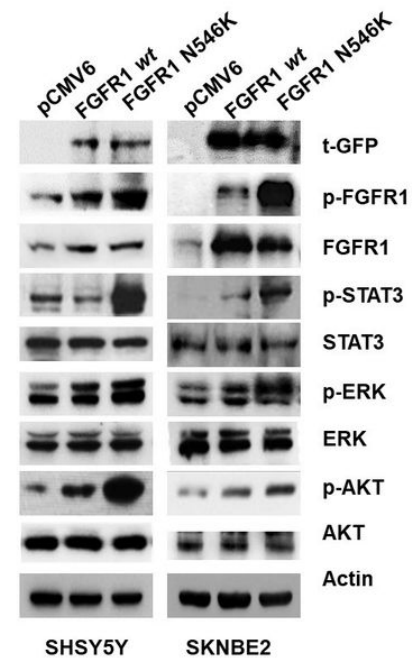

C

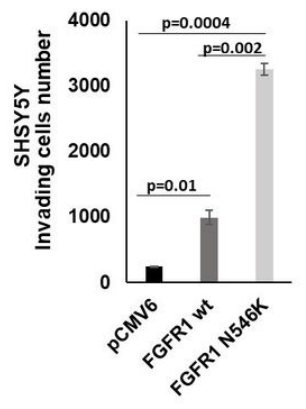

B
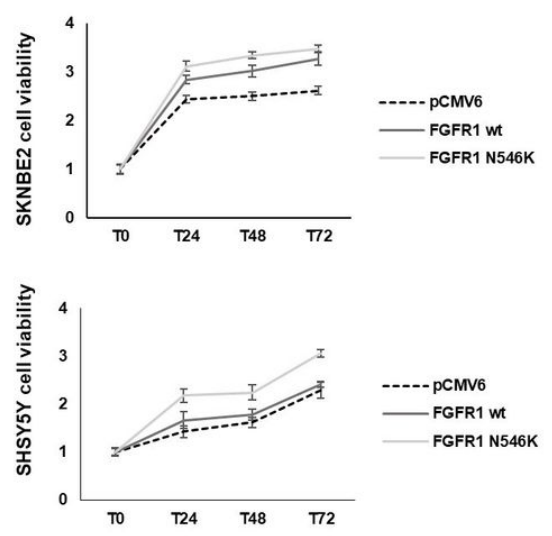

D
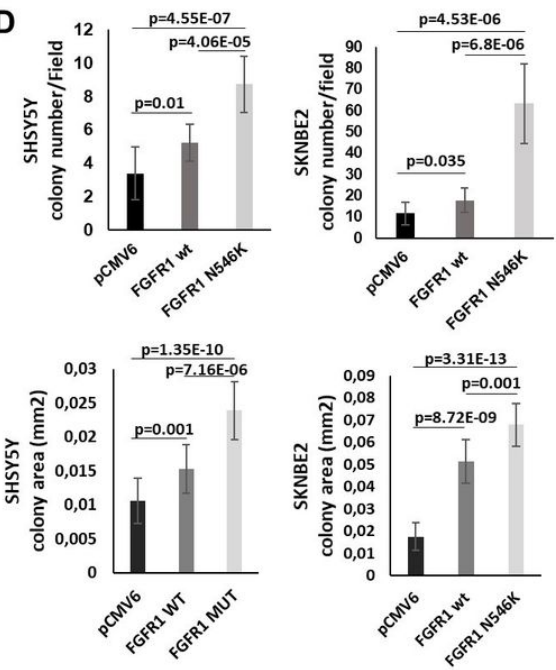

\section{Figure 4}

FGFR1 ${ }^{\text {wt }}$ and FGFR1 ${ }^{\text {N546K }}$ protein overexpression in NB cells. SH5YSY and SKNBE2 cells were transiently transfected with pCMV6-empty vector, pCMV6FGFR1 ${ }^{\mathrm{wt}}$, pCMV6-FGFR1 ${ }^{\mathrm{N} 546 \mathrm{~K}}$. (A) Total protein extracts were analyzed by western blotting to evaluate the levels of phosphorylated and total FGFR1, STAT3, ERK, and-AKT. The t-GFP and $\beta$-Actin protein levels were used as trasfection control and loading control, respectively. (B) Cell viability in FGFR ${ }^{\mathrm{wt}}$ and FGFR1 ${ }^{N 546 K}$ overexpressing cells is shown as fold change respect to the control (pCMV6). (C) Invading cells, (D) colony number and area were analyzed. $\mathrm{mm}^{2}=$ square millimetres. $\mathrm{p}=p$-value. 


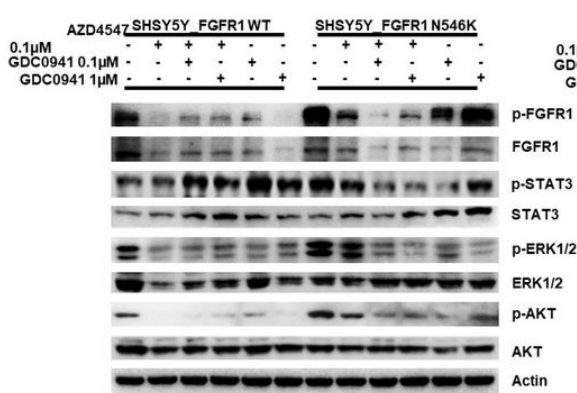

AZD454 EKNEBE2_FGFR1WT

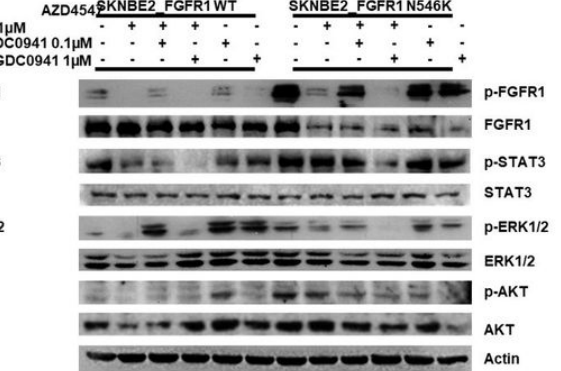

C
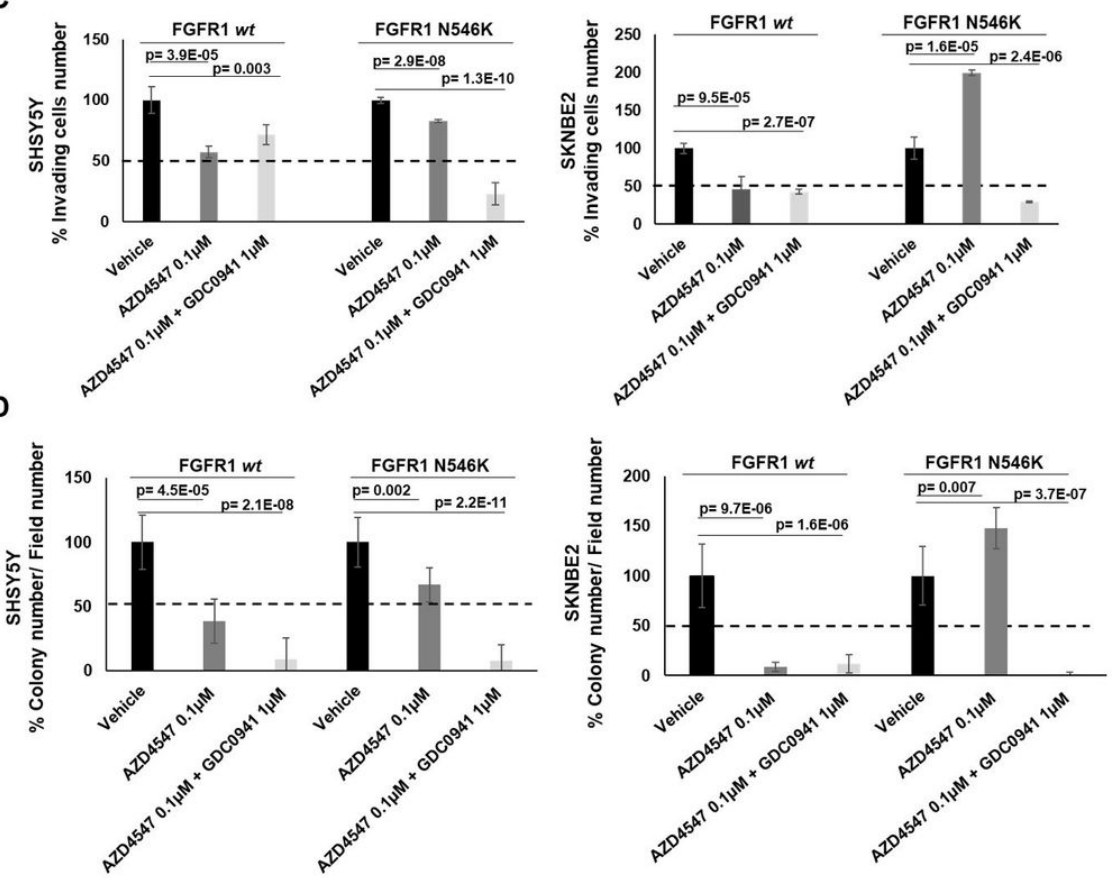

Figure 5

Targeting of FGFR1 signaling by combination treatment with AZD4547 and GDC0941. SH5YSY and SKNBE2 cells were transiently transfected with pCMV6FGFR1 ${ }^{\text {wt }}$, pCMV6- FGFR1 ${ }^{\text {N546K }}$ and pCMV6 empty vector. (A, B) Total protein extracts were analyzed by western blotting to evaluate the levels of phosphorylated and total-FGFR1, STAT3, ERK and AKT. The $\beta$-Actin protein levels were used as loading control. (C) The ability of cells treated with single AZD4547 0.1 $\mu \mathrm{M}$ and with combination of AZD4547 $0.1 \mu \mathrm{M}$ and GDC0941 $1 \mu \mathrm{M}$ to invade and migrate through a matrigel-coated membrane support was evaluated. The number of invading FGFR1 ${ }^{\text {wt }}$ or FGFR1 ${ }^{\text {N546K }}$ overexpressing cells are shown in percentage respect to untreated cells (100\% vehicle). (D) The ability of cells to form neuropheres after treatment with AZD4547 $0.1 \mu \mathrm{M}$ alone and in combination with GDC0941 $1 \mu \mathrm{M}$ was evaluated. The colony number of FGFR $1^{\text {wt }}$ or FGFR $1^{\text {N546K }}$ overexpressing cells are shown in percentage respect to untreated cells $(100 \%$ vehicle $)$. Vehicle $=\mathrm{DMSO} p=p$-value

\section{Supplementary Files}

This is a list of supplementary files associated with this preprint. Click to download.

- supplementaryFGFR1.docx 